4. Badran AM, Vahedi K, Berrebi D, Catana D, De Lagausie P, Drouet L et al. Pediatric ampullar and small bowel blue rubber bleb nevus syndrome diagnosed by wireless capsule endoscopy. J Pediatr Gastroenterol Nutr. 2007; 44: 283-6.

5. De Bona M, Bellumat A, De Boni M. Capsule endoscopy for the diagnosis and follow-up of blue rubber bleb nevus syndrome. Dig Liver Dis. 2005; 37: 451-3.

6. Agnese M, Cipolletta L, Bianco MA, Quitadamo P, Miele E, StaianoA.Blue rubber bleb nevus syndrome.ActaPaediatr. 2010;99:632-5.

7. Yuksekkaya H, Ozbek O, Keser M, Toy H. Blue rubber bleb nevus syndrome: successful treatment with sirolimus. Pediatrics. 2012;129:e1080-e1084.

8. Gonzalez D, Elizondo BJ, Haslag S, Buchanan G, Burdick JS, Guzzetta PC et al.Chronic subcutaneous octreotide decreases gastrointestinal blood loss in bluerubber-bleb nevus syndrome.JPediatr Gastroenterol Nutr. 2001; $33: 183-8$.

\section{Synchronous cholangiocarcinoma and gall bladder carcinoma}

The simultaneous occurrence of malignancies in the bile duct and the gall bladder is not frequently reported. They might be arising as a part of the spectrum of rare synchronous malignancies or could be occurring as a result of local spread or metastasis. When considering a diagnosis of synchronous primaries, the criteria that have been used to support this relatively rare occurrence must be taken into account since the two sites are anatomically close to each other and have embryologic similarities as well. Gertsch et al have attempted to describe the criteria to differentiate between the two entities. ${ }^{1}$ The diagnosis of these synchronous tumours is rarely made preoperatively. They should be suspected and looked for in every instance by intraoperative endoscopy and meticulous histologic examination of the gall bladder as they are associated with fairly good prognosis if complete surgical resection is possible. Here, we report a rare case of synchronous cholangiocarcinoma and gall bladder carcinoma in a 35 year old female.

\section{Case Report}

A 35-year old female presented to the OPD with pain abdomen for the past 4 months. Her blood work-up revealed raised values of bilirubin (total bilirubin-7.9 $\mathrm{mg} / \mathrm{dl})$ and alkaline phosphate (720 IU/L). Computed Tomography showed a mass measuring $2.7 \mathrm{~cm} \times 2.5 \mathrm{~cm}$ at the lower end of the common bile duct with cholelithiasis. The patient was taken for surgery, wherein a growth was found in the lower part of the common bile duct with dense adhesions over Calot's triangle, the porta hepatis and the gall bladder. An extended cholecystectomy with extrahepatic common bile duct excision and pancreaticoduodenectomy with a radical lymphadenectomy was performed.

On opening the common bile duct, gross examination revealed a grey-white growth measuring $1.2 \mathrm{~cm} x 1 \mathrm{~cm} \times 1 \mathrm{~cm}$ was present at the resected end encircling it. Cut section of the gall bladder showed wall thickening in the fundus region measuring $1 \mathrm{~cm}$.

Histological examination showed a moderately differentiated adenocarcinoma of the gall bladder (Figure 1) and the common bile duct (Figure 3) with extension beyond the serosa and focally involving the pancreas and the muscle layer of the duodenum. The adjacent mucosa of both showed in situ changes (Figure 2) without any direct continuity (Figure 4) between the two tumors. Solitary lymph node metastasis was also present. Immunohistochemistry was done wherein both the gall bladder carcinoma and the cholangiocarcinoma showed more than 10 per cent Ki67 positivity and absence of $\mathrm{p} 53$ mutation.

\section{Discussion}

Cholangiocarcinoma is an uncommon tumour seen mainly in the elderly. The synchronous occurrence of 


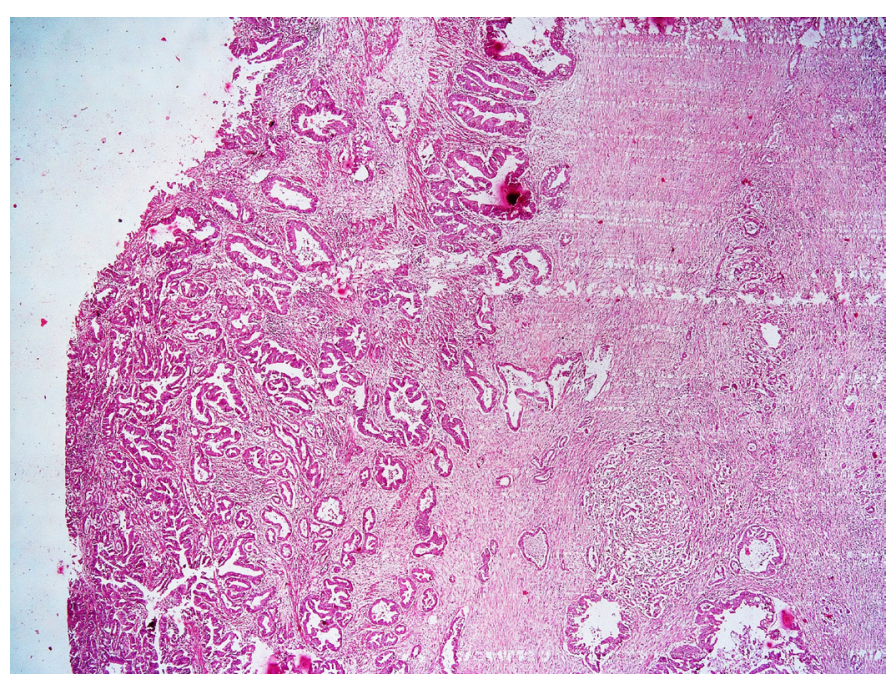

Figure 1: 20x, H\&E, Gall bladder carcinoma

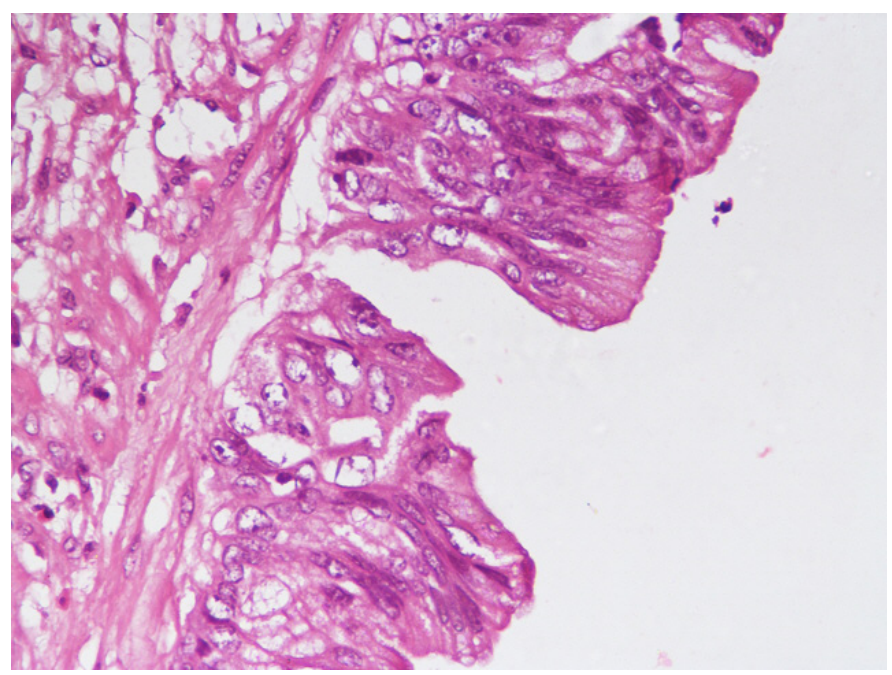

Figure 2: 400x, H\&E, Gall bladder with carcinoma in situ

gall bladder cancer with bile duct cholangiocarcinoma is rarely encountered. ${ }^{2}$ This is probably due to insufficient sampling of the gall bladder when performing resections for extrahepatic bile duct malignancies. Most of these cases have been reported from Japan where the incidence is approximately 5 to 7 per cent. ${ }^{3}$ A large proportion of these malignancies are associated with an anomalous pancreatic-bile duct junction (APBDJ). ${ }^{4}$ In patients without APBDJ, gall bladder carcinomas follow the

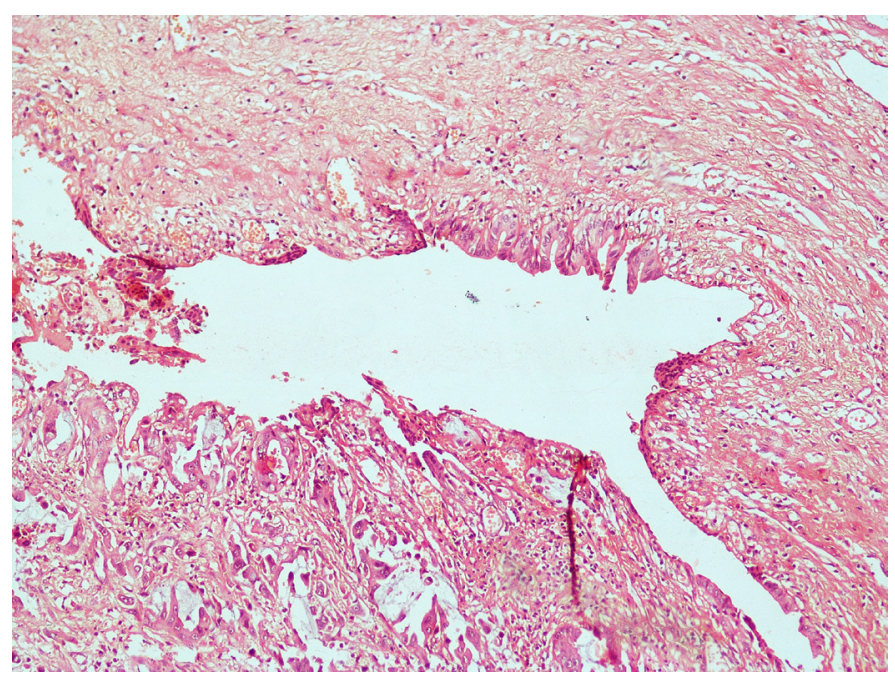

Figure 3: 100x, H\&E, Common bile duct carcinoma with transformation zone

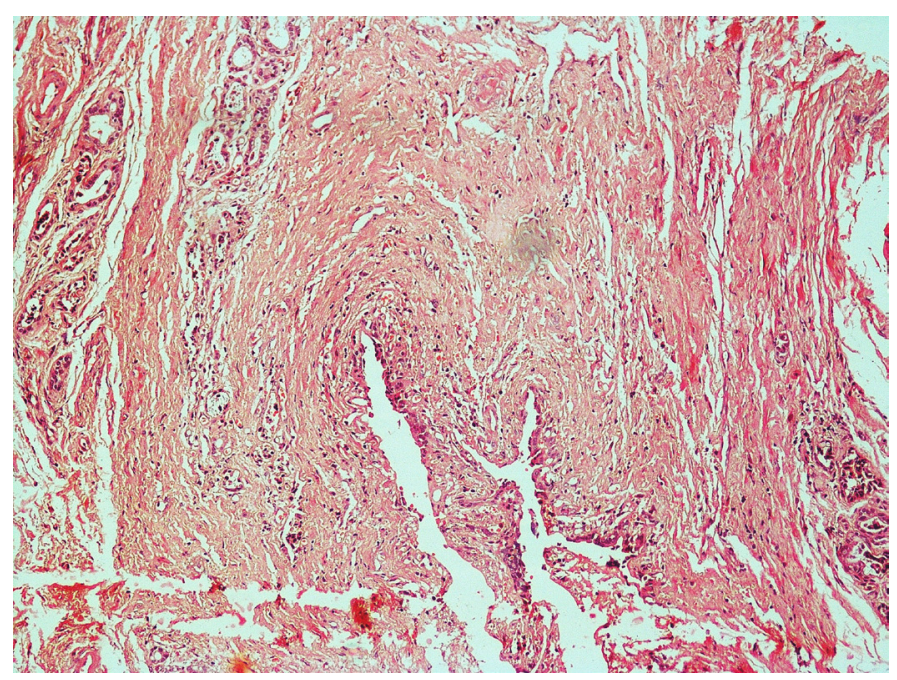

Figure 4: 100x, H\&E, Intervening normal common bile duct

dysplasia- in situ- invasive carcinoma sequence rather than the adenoma-carcinoma sequence. ${ }^{5}$ This clinical condition is often confused with metastasis from a primary elsewhere in the biliary tree. Gertsch et al have advocated that the best way to differentiate between the two entities is by applying the following criteria:

1) no direct continuity between the two tumors

2) a growth pattern typical of a primary tumour and 
3) clear histologic differences between the two tumors, as in our case.

The criteria for differentiating metastasis and synchronous primaries are still being formulated. ${ }^{6}$ Kurosaki et al have proposed a mapping technique to distinguish between the two lesions. ${ }^{2}$ Over the past few years, multifocal origins have been considered for such synchronous tumours. This may also occur due to intraepithelial spread, although the incidence is only 4 percent. $^{6}$

The concept of field cancerisation was first introduced by Slaughter in 1953 to explain the possibility of distant related primary tumours in the upper aerodigestive tract. ${ }^{7}$ Unfortunately, this theory has seldom been applied to cases of extrahepatic biliary tree malignancies. As the biliary tree is constantly exposed to bile, it could be involved in carcinogenesis especially in patients with APBDJ. ${ }^{8}$ This concept has been supported by findings of high levels of secondary bile acids in the bile of patients with gall bladder cancer.

p53 gene mutations have been used to determine the clonality. ${ }^{7}$ Mutations in p53 are seen in 58\% to $92 \%$ of all gall bladder cancers and have also been found at histologically normal- appearing epithelium near areas with gallbladder cancer. ${ }^{9}$ Hori et al stated that more studies on p53 mutations would be needed in synchronous malignancies unrelated to APBDJ. ${ }^{6}$

Cases of synchronous tumours which have not yet metastasized to distant organs should be treated with radical resection if feasible as this is associated with a fairly good prognosis. ${ }^{2}$ On the other hand, metastatic disease, involvement of the cystic duct and perineural invasion have been associated with poor prognoses.

The simultaneous occurrence of multiple tumors of the extra-hepatic biliary tract may be more common than previously thought and the incidence of unsuspected gallbladder carcinoma in association with bile duct carcinoma may be quite high. In this case, the preoperative diagnosis was one of cholangiocarcinoma with cholelithiasis; however, radical resection with careful microscopic examination enabled it to be diagnosed successfully.
PREETHI SEKAR NEELAM SOOD

Department of Pathology, D.D.U. Hospital, Harinagar, New Delhi, India

Correspondence: Dr Preethi Sekar Email:drpreethisekar@gmail.com

\section{References}

1. Gertsch P, Thomas P, Baer H, Lerut J, Zimmermann A, Blumgart LH. Multiple tumors of the biliary tract. Am J Surg. 1990;159:386-8.

2. Kurosaki I, Watanabe H, Tsukada K, Hatekayama K. Synchronous primary tumors of the extrahepatic bile duct and gallbladder. J Surg Oncol.1997;65:258-62.

3. P. J. Shukla, S. G. Barreto, S. V. Shrikhande, M. R. Ramadwar, K. K. Deodhar, S. Mehta, et al. Simultaneous gallbladder and bile duct cancers: revisiting the pathological possibilities. 2008; 10:48-53.

4. Nakao A, Sakagami K, Uda M, Mitsuoka S. Double cancers of the gallbladder and bile duct with anomalous choledochopancreatic duct junction. J Gastroenterol. 1997;32:110-3.

5. Wistuba II, Miguel JF, Gazdar AF, Albores-Saavedra J. Gallbladder adenomas have molecular abnormalities different from those present in gallbladder carcinomas. Hum Pathol.1999;30:21-5.

6. Hori H, Ajiki T, Fujita T, Okazaki T, Suzuki Y, Kuroda $\mathrm{Y}$, et al. Double cancer of gall bladder and bile duct not associated with anomalous junction of the pancreatobiliary duct system. Jpn J Clin Oncol. 2006;36:638-42.

7. Ha PK, Califano JA. The molecular biology of mucosal field cancerization of the head and neck. Crit Rev Oral Biol Med. 2003;14:363-9.

8. Strom BL, Soloway RD, Rios-Dalenz JL, RodriguezMartinez HA, West SL, Kinman JL et al. Biochemical epidemiology of gallbladder cancer. Hepatology. 1996;23:1402-11.

9. Wistuba II, Sugio K, Hung J, Kishimoto Y, Virmani AK, Roa I, et al. Allele-specific mutations involved in the pathogenesis of endemic gallbladder carcinoma in Chile. Cancer Res. 1995;55:2511-5. 